\title{
Pretreatment quality assurance of volumetric modulated arc therapy on patient CT scan using indirect 3D dosimetry system
}

\author{
Shanmugam Thirumalai Swamy ${ }^{1,2}$, Chandrasekaran Anuradha ${ }^{2}$, Murugesan Kathirvel1, \\ Gandhi Arun', Shanmuga Subramanian ${ }^{1}$
}

${ }^{1}$ Department of Radiation Oncology, Yashoda Hospitals, Hyderabad, India

${ }^{2}$ School of Advanced Sciences, VIT University, Vellore, India

Received August 29, 2014; Revised October 16, 2014; Accepted October 18, 2014; Published Online October 22, 2014

\section{Original Article}

\begin{abstract}
Purpose: Aim of this study is to clinically implement the COMPASS 3D dosimetry system for pretreatment quality assurance of volumetric modulated arc therapy (VMAT-RapidArc) treatment plans. Methods: For this study, 10 head and neck (H\&N) and pelvis VMAT plans dose response from Linac was measured using COMPASS system along with MatriXX ${ }^{\text {Evolution }}$ and 3D dose reconstructed in the patient computed tomography (CT) scan. Dose volume histograms and 3D gamma were used to evaluate difference between the measured and calculated values. In order to validate the COMPASS system, dose response for open fields were acquired for both homogeneous and inhomogeneous phantoms. Results: The average dose difference between Eclipse treatment planning system (TPS) calculated and COMPASS measured (homogenous medium) in normalization region, inner region, penumbra region and buildup region was less than $\pm 2 \%$. In inhomogeneous phantom, there was a maximum difference $-3.17 \%$ in lung, whereas the difference other densities was within $\pm 2 \%$. The systematic increase in the average 3D gamma between the TPS calculated and COMPASS measured for VMAT plans with known dose errors and multi-leaf collimator (MLC) offset errors shows that COMPASS system was sensitive enough to find clinical significant errors. The 3D dose parameters (D95, D1, and average dose) of all H\&N and pelvis patients were well within the clinically acceptable tolerance level of $\pm 5 \%$. The average 3D gammas for planning target volumes (PTV) and organ at risks (OAR) of the patients were less than 0.6. Conclusion: The results from this study show that COMPASS along with MatriXX ${ }^{\text {Evolution }}$ can be effectively used for pretreatment verification of VMAT plans in the patient anatomy.
\end{abstract}

Keywords: COMPASS; VMAT; 3D Gamma; Pretreatment QA

\section{Introduction}

In advanced radiotherapy techniques, each patient's treatment plan is customized, to produce high gradient dose distribution to the target and low dose to the critical organs. Volumetric Modulated Arc Therapy (VMAT- RapidArc) produces highly conformal dose distribution by simultaneously changing multi-leaf collimator (MLC) position, dose rate and gantry speed during patient treatment. ${ }^{1-3}$ Complex treatment deliveries demand a comprehensive quality assurance (QA) procedure. American Association of Physicists in Medicine Task Group (AAPM TG) $-82^{4}$ recommends verification of intensity modulated treatment plans with an independent QA method before treatment delivery. Traditionally pretreatment QA's are performed in a phantom using ion chamber, film, 2D array, and electronic portal imaging device (EPID). Each of these devices has been proven useful but has its own limitations. ${ }^{5-11}$ Position of ion chamber in the high dose gradient area leads to discrepancy between meas- ured and the treatment planning system (TPS) calculated dose. Film shows excellent spatial resolution; however, problems like chemical processing, scanner readout, and time delay exists. A 2D array shows the dose distribution immediately after the treatment delivery, but their results have limited resolution. Also, results provided by them cannot be directly used to identify delivery errors in tumor and normal tissues. Benjamin et al. ${ }^{12}$ showed that there is lack of correlation between gamma passing rates from 2D array and dose differences in critical anatomic regions of interest. To address this issue, alternate QA techniques has been developed to verify the 3D dose distribution by measuring fluence at different gantry angle using ion chamber matrix or EPID in a patient computed tomography (CT) scan. ${ }^{13-18}$ The COMPASS QA system (IBA Dosimetry, Germany) is one such technique which uses MatriXX ${ }^{\text {Evolution }}$ along with gantry 
angle sensor. It consists of (i) a measurement-based dose reconstruction, and (ii) model-based dose calculation.

\section{Measurement based dose reconstruction}

(i) Detector dose response is predicted from the patient treatment plan parameters. (ii) Measured dose response from the Matrix ${ }^{\text {Evolution }}$ is then compared with predicted dose response. (iii) The difference between the predicted and measured response along with correction kernel was used to derive the reconstruction fluence. Finally reconstructed fluence is fed to the dose engine based on collapsed cone model for computation of $3 \mathrm{D}$ dose within the patient CT scan. The dose calculated from the reconstructed fluence is referred as "indirectly measured" (COMPASS measured).

\section{Model based dose calculation}

COMPASS system can compute dose in patient CT scan using inbuilt beam model. The purpose of the dose computation is to provide an independent cross-verification of TPS calculated dose.

In addition, COMPASS has a facility to compare the 3D dose distribution and dose volume histograms (DVH) between measured and TPS calculated. Previous studies have validated the dose reconstruction method of COMPASS system for fixed field IMRT against film based QA in phantoms. ${ }^{13,15,17}$ Ramesh et al. ${ }^{14}$ has shown the experimental validation of COMPASS system for rotational IMRT in inhomogeneous phantom. This study focuses on pretreatment QA of VMAT treatment plan on "measurement based dose reconstruction". We have evaluated the performance of COMPASS 3D dosimetry system clinically: (i) In open field sizes $\left(10 \times 10 \mathrm{~cm}^{2}\right.$ and $20 \times 20 \mathrm{~cm}^{2}$ ) to evaluate the accuracy of beam modeling both in homogeneous and inhomogeneous media, (ii) To check the accuracy of the system in detecting MLC and dose errors and (iii) To implement the system for VMAT patient specific QA.

\section{Methods and Materials}

All measurements were performed using $6 \mathrm{MV}$ photon beam from dual energy Clinac-iX (Varian Medical Systems, Palo Alto, USA). The machine was equipped with millennium 120 MLC, on-board imager (OBI) and maximum dose rate of 600 $\mathrm{MU} / \mathrm{min}$. Conventional pretreatment QA for VMAT plans were performed with point dose measurements and $2 \mathrm{D}$ planar dosimetry using $0.13 \mathrm{cc}$ ionization chamber in a cube phantom and MatriXX ${ }^{\text {Evolution }}$ in multicube phantom (IBA Dosimetry) respectively. MatriXX ${ }^{\text {Evolution }}$ contains 1020 parallel plane ion chambers (32 $\times 32$ matrix) with an active area of $24.4 \times 24.4 \mathrm{~cm}^{2}$ having $7.62 \mathrm{~mm}$ resolution at isocenter of 100 $\mathrm{cm}$. In point dose measurement, percentage dose variation was calculated between the measured dose using ion chamber and the calculated dose in Eclipse TPS. For 2D planar dosimetry (frontal plane), global gamma analysis was per- formed using OmniPro Im'RT software with criteria of 3\% dose difference (DD) and 3mm distance to agreement (DTA).

In this study, COMPASS (V2.1) was used as a pretreatment 3D QA tool to validate VMAT plans. Dose response from treatment plans was measured using MatriXX ${ }^{\text {Evolution }}$ with 5 cm RW3 buildup plates and gantry angle sensor placed on a gantry holder mount, Figure 1 (source to detector distance of $76.2 \mathrm{~cm}$ ). The dose calculation engine in the COMPASS system is collapsed cone convolution/superposition (CCC/S) algorithm, whereas Eclipse TPS uses Analytical Anisotropic Algorithm (AAA). Since these two algorithms use different approach in dose calculation, simple open field sizes were first validated in both homogeneous and inhomogeneous mediums.

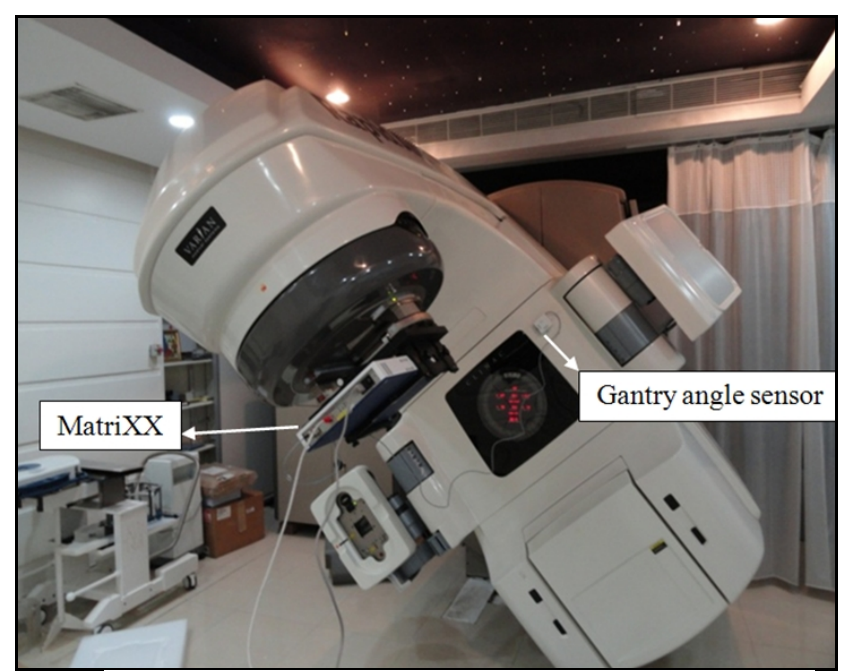

FIG.1: Setup for the pretreatment quality assurance for VMAT treatment delivery. MatriXX ${ }^{\text {Evolution }}$ with $5 \mathrm{~cm}$ RW3 buildup plates and gantry angle sensor placed on a gantry holder mount with source to detector distance- $76.2 \mathrm{~cm}$.

\section{Open field measurements}

To verify the accuracy of beam modeling and algorithm difference, the fluence for MLC defined open fields (ranging from $5 \times 5 \mathrm{~cm}^{2}$ to $25 \times 25 \mathrm{~cm}^{2}$ ) were acquired by COMPASS system on a homogeneous phantom. Average dose calculated and measured by Eclipse TPS and COMPASS QA system respectively were compared at normalization, inner, outer, penumbra and buildup regions as per AAPM TG-53 ${ }^{19}$ recommendations (Figure 2a).

Average dose had been compared for different Hounsfield unit (HU) mediums teflon (990 HU), derlin (340 HU), acrylic (120 HU), polystyrene (-35 HU), LDPE (-100 HU), PMP $(-200 \mathrm{HU})$ and air $(-1000 \mathrm{HU})$ in CAT Phantom (Figure $2 \mathrm{~b})$. This average dose comparison was done for two sets of MLC defined open fields $\left(10 \times 10 \mathrm{~cm}^{2}\right.$ and $\left.20 \times 20 \mathrm{~cm}^{2}\right)$ in an inhomogeneous phantom. 

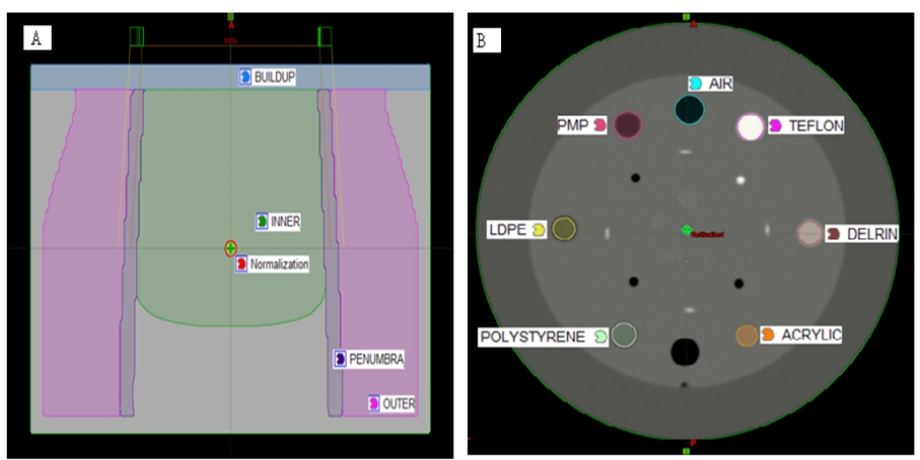

FIG.2 (a): Homogeneous phantom created in Eclipse TPS as per AAPM TG-53 recommendations. Based on beam geometry, phantom was broken into different regions to analyze the agreement between TPS calculated and COMPASS measured dose. FIG. 2(b): Transverse view of CAT phantom CT scan with different HU medium.

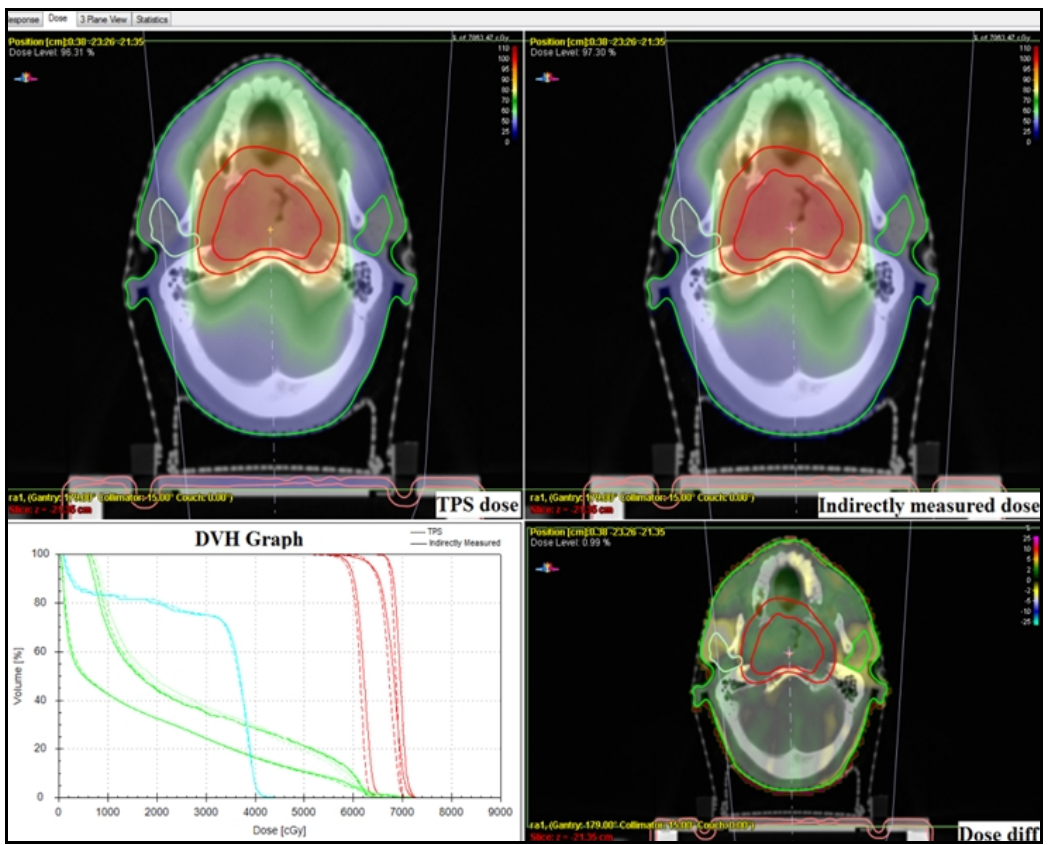

FIG. 3: Dose distribution and DVH comparison between Eclipse TPS calculated and COMPASS measured for an H\&N patient.

\section{Intentional error measurements}

To validate the accuracy of the COMPASS system, VMAT plans with known dose error of \pm 3 and $\pm 5 \%$ and MLC errors of 1,3 and $5 \mathrm{~mm}$ due to one side of MLC bank mis-calibration were delivered. For 2D planar dosimetry, dose plane from TPS plan without error was used as reference in OmniPro Im'RT software for gamma analysis. For 3D dosimetry, VMAT plan without error was exported to COMPASS system.

\section{Patient plan evaluation}

10 head \& neck $(\mathrm{H} \& \mathrm{~N})$ and 10 pelvis VMAT plans with two full arcs $\left(181^{\circ}-179^{\circ}\right.$ clock wise / counter clock wise) were chosen for this study. The H\&N patients contain three planning target volumes (PTV) with different level of dose prescription (simultaneous integrated boost, Figure 3 ) and single PTV for pelvis patients. VMAT plans were optimized in
Eclipse TPS (version 8.9) using Progressive Resolution Optimizer -II and final dose calculations were performed using AAA with $2.5 \mathrm{~mm}$ grid resolution. For pretreatment 3D QA, these VMAT plans along with patient's CT scan, structure set and 3D dose planes were exported to COMPASS in DICOM RT format.

On treatment machine, MatriXX ${ }^{\text {Evolution }}$ (Trigger Mode) was used for measuring dose response. COMPASS system calculates predicted dose response using DICOM RT plan parameters (gantry angle, MLC position, MU, etc) from TPS, detector model and in-built beam model. This predicted dose response was compared against the corresponding measured dose response and the difference was incorporated in dose calculation (Figure 4). The final dose distribution was reconstructed on patient CT using CCC/S algorithm with same grid size of $2.5 \mathrm{~mm}$. The average doses for PTV's and organs 
at risk (OAR's) in H\&N as well as pelvis patients were compared between TPS calculated and COMPASS measured. Dose to $95 \%$ of the PTV volume (D95) and max dose to spinal cord (D1) was also evaluated. The average 3D global gamma was calculated using criteria of $3 \mathrm{~mm}$ DTA and $3 \%$ DD.

\section{Results}

\section{Open fields}

The percentage difference of average dose between TPS calculated and COMPASS measured for different region and HU material were shown in Figure 5a and 5 b respectively. In order to understand the dose difference arising from the beam modeling, the open field measurements were analyzed. Percentage difference of average dose for all open fields in normalization, inner, penumbra and buildup regions was less than AAPM TG-53 ${ }^{19}$ recommended value $0.5 \%, 1.5 \%, 10 \%$ $(2 \mathrm{~mm})$ and $20 \%$ respectively. For outer region suggested value was $2 \%$, but in our study due to low dose a maximum difference of $8.3 \%$ was observed.

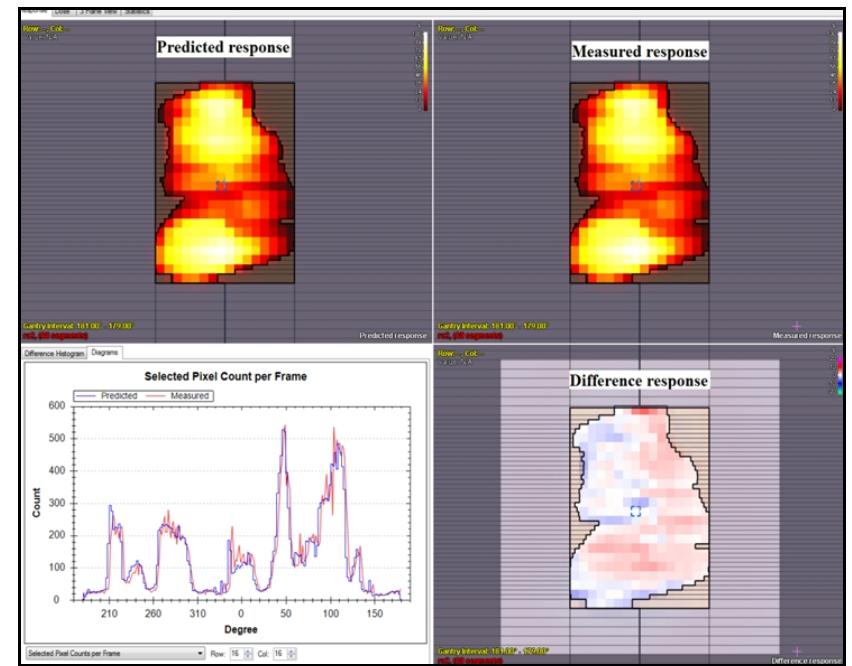

FIG. 4: The predicted dose response by COMPASS system was compared against the corresponding measured dose response from Ma-

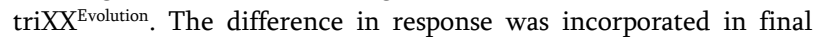
dose reconstruction.

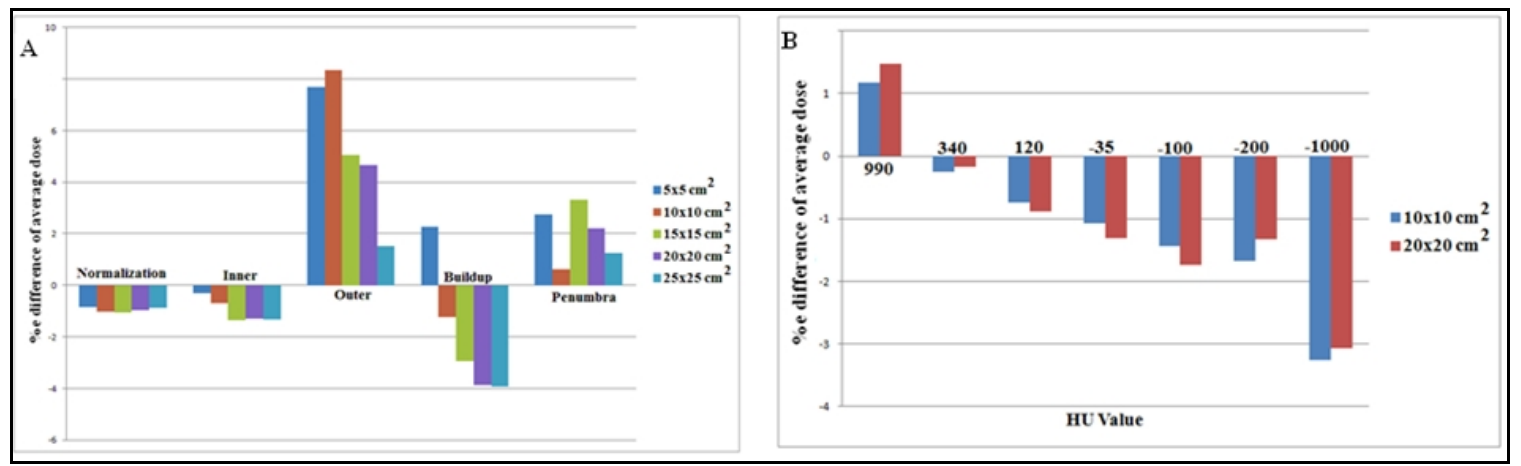

FIG. 5 (a): Percentage difference in average dose for different regions of interest in homogeneous phantom for MLC defined open fields size of 5 $\times 5 \mathrm{~cm}^{2}, 10 \times 10 \mathrm{~cm}^{2}, 15 \times 15 \mathrm{~cm}^{2}, 20 \times 20 \mathrm{~cm}^{2}$ and $25 \times 25 \mathrm{~cm}^{2}$ respectively. FIG. 5 (b): Percentage difference in average dose for different HU material in CAT phantom for MLC defined open fields $10 \times 10 \mathrm{~cm}^{2}$ and $20 \times 20 \mathrm{~cm}^{2}$

\section{Intentional errors}

Table 1 shows the gamma agreement index (GAI) for known dose and MLC errors between TPS calculated and MatriXX ${ }^{\text {Evolution }}$ measured in multicube phantom. GAI is defined as percentage of points passing the gamma evaluation criteria. COMPASS system provides the clinical relevance of dose and MLC errors by calculating the average dose difference and 3D gamma for PTV, rectum and bladder.

TABLE 1: Shows gamma agreement index (GAI) for measurements with intentional dose and MLC errors, whereas COMPASS system provides the clinical relevance of errors by calculating the average 3D gamma and dose difference for PTV, rectum and bladder.

\begin{tabular}{cccccccc}
\hline \hline \multirow{2}{*}{ Description } & \multirow{2}{*}{ GAI } & \multicolumn{3}{c}{ average 3D gamma } & \multicolumn{3}{c}{ \% difference in average dose } \\
\cline { 3 - 9 } & & PTV & Rectum & Bladder & PTV & Rectum & Bladder \\
\hline Without Error & 97.5 & 0.33 & 0.28 & 0.25 & -0.73 & 3.11 & -2.77 \\
3\% Dose Error & 94.8 & 1.03 & 0.27 & 0.55 & -3.80 & 0.56 & -5.28 \\
5\% Dose Error & 89.9 & 1.50 & 0.38 & 0.69 & -5.64 & -2.66 & -6.96 \\
-3\% Dose Error & 92.4 & 0.58 & 0.40 & 0.21 & 2.04 & 5.22 & 0.58 \\
-5\% Dose Error & 87.3 & 1.22 & 0.61 & 0.45 & 4.39 & 7.62 & 2.81 \\
1mm MLC Error & 94.3 & 0.45 & 0.28 & 0.5 & -1.06 & 2.61 & -6.53 \\
3mm MLC Error & 92.95 & 0.77 & 0.45 & 0.41 & -1.61 & 2.18 & 3.33 \\
5mm MLC Error & 85.65 & 1.08 & 0.61 & 0.78 & -0.96 & 1.30 & 7.93 \\
\hline \hline
\end{tabular}


TABLE 2: 3D gamma and dose volume difference between Eclipse TPS calculated and COMPASS measured for 10 H\&N patients PTV's and OAR'S. For same patients, point dose difference from ion chamber and 2D gamma from planar dosimetry were listed.

\begin{tabular}{|c|c|c|c|c|c|c|c|c|c|c|c|c|c|}
\hline Parameters & Structure & Pt-1 & Pt-2 & Pt-3 & Pt-4 & Pt-5 & $\mathrm{Pt}-6$ & Pt-7 & Pt-8 & Pt-9 & Pt-10 & Mean & ${ }_{ \pm} \mathrm{SD}$ \\
\hline \multirow{4}{*}{$\begin{array}{l}\text { \% of point dose difference } \\
\text { gamma agreement index }\end{array}$} & & 0.97 & 1.07 & -1.11 & -0.67 & -0.93 & -1.29 & -2.60 & 2.09 & -2.45 & 0.63 & -0.43 & 1.56 \\
\hline & & 96.1 & 97.0 & 97.9 & 95.1 & 96.7 & 95.5 & 97.9 & 98.3 & 98.1 & 95.1 & 96.7 & 1.26 \\
\hline & PTV1 & -2.02 & -1.53 & -2.07 & -2.46 & -2.09 & -1.94 & -1.18 & -1.25 & -0.79 & -2.97 & -1.83 & 0.65 \\
\hline & PTV2 & -2.36 & -1.47 & -1.98 & -2.69 & -2.08 & -1.36 & -1.41 & -1.38 & -0.96 & -2.42 & -1.81 & 0.57 \\
\hline \multirow{6}{*}{$\begin{array}{c}\% \text { difference in average } \\
\text { dose }\end{array}$} & PTV3 & -2.56 & -0.35 & -1.62 & -1.88 & -2.91 & -1.37 & -2.62 & -2.65 & -1.65 & -2.71 & -2.03 & 0.81 \\
\hline & Rt Parotid & 0.71 & -4.00 & -3.93 & -1.89 & 4.74 & -3.10 & -2.75 & 2.38 & -2.05 & -4.52 & -1.44 & 3.07 \\
\hline & Lt Parotid & -2.42 & -2.69 & 0.33 & 3.76 & 3.13 & -2.50 & 1.23 & -0.51 & -1.28 & 3.99 & 0.30 & 2.62 \\
\hline & Spinal cord & -2.95 & -1.12 & -2.87 & -3.17 & -0.83 & -1.49 & 0.23 & -1.23 & -0.57 & -1.18 & -1.52 & 1.13 \\
\hline & PTV1 & 0.37 & 0.33 & 0.49 & 0.31 & 0.41 & 0.57 & 0.40 & 0.36 & 0.27 & 0.44 & 0.40 & 0.09 \\
\hline & PTV2 & 0.39 & 0.30 & 0.45 & 0.36 & 0.44 & 0.41 & 0.36 & 0.33 & 0.27 & 0.48 & 0.38 & 0.07 \\
\hline \multirow{5}{*}{ average $3 \mathrm{D}$ gamma } & PTV3 & 0.43 & 0.24 & 0.30 & 0.41 & 0.46 & 0.52 & 0.46 & 0.56 & 0.40 & 0.54 & 0.43 & 0.10 \\
\hline & Rt Parotid & 0.14 & 0.30 & 0.08 & 0.22 & 0.25 & 0.40 & 0.22 & 0.11 & 0.13 & 0.30 & 0.22 & 0.10 \\
\hline & Lt Parotid & 0.31 & 0.26 & 0.18 & 0.22 & 0.29 & 0.28 & 0.14 & 0.09 & 0.11 & 0.14 & 0.20 & 0.08 \\
\hline & Spinal cord & 0.39 & 0.11 & 0.36 & 0.21 & 0.20 & 0.37 & 0.24 & 0.15 & 0.11 & 0.18 & 0.23 & 0.11 \\
\hline & PTV1 & -1.95 & -2.83 & -1.80 & -2.22 & -2.28 & -1.05 & -0.26 & -0.91 & -0.90 & -2.50 & -1.67 & 0.84 \\
\hline \multirow[t]{2}{*}{$\%$ difference in D95 } & PTV2 & -2.29 & -1.10 & -1.72 & -2.23 & -2.36 & 0.29 & -0.99 & -1.80 & -0.52 & -2.24 & -1.50 & 0.89 \\
\hline & PTV3 & -2.20 & 0.86 & -0.23 & -0.84 & -2.56 & 1.85 & -1.35 & -2.68 & -0.16 & -2.33 & -0.96 & 1.54 \\
\hline$\%$ difference in D1 & Spinal cord & -1.88 & -1.00 & -3.17 & -1.14 & -1.37 & -2.84 & -0.27 & -3.60 & 0.85 & -1.96 & -1.64 & 1.36 \\
\hline
\end{tabular}

TABLE 3: 3D gamma and dose volume difference between Eclipse TPS calculated and COMPASS measured for 10 pelvis patients.

\begin{tabular}{|c|c|c|c|c|c|c|c|c|c|c|c|c|c|}
\hline Parameters & Structure & Pt-1 & $\mathrm{Pt}-2$ & Pt-3 & Pt-4 & Pt-5 & $\mathrm{Pt}-6$ & $\mathrm{Pt}-7$ & Pt-8 & Pt-9 & Pt-10 & Mean & $\pm \mathrm{SD}$ \\
\hline$\%$ of point dose difference & & 0.16 & 2.71 & 0.32 & 1.6 & -0.91 & 0.1 & 0.93 & 0.16 & -1.07 & -1.11 & 0.29 & 1.22 \\
\hline gamma agreement index & & 98.4 & 97.2 & 98.6 & 99.1 & 95.1 & 96.4 & 97.7 & 96.3 & 97.0 & 97.9 & 97.4 & 1.22 \\
\hline \multirow{4}{*}{$\begin{array}{c}\% \text { difference in average } \\
\text { dose }\end{array}$} & PTV & -1.82 & -1.27 & -0.42 & -0.83 & -1.29 & -1.52 & -0.16 & -0.60 & -1.49 & 0.82 & -0.86 & 0.79 \\
\hline & Rectum & 0.43 & 0.50 & 1.64 & 2.35 & 0.40 & 0.18 & 3.49 & 0.39 & -3.45 & 1.13 & 0.71 & 1.81 \\
\hline & Bladder & -3.75 & -1.97 & 0.02 & -2.03 & -1.93 & -1.63 & -2.38 & -2.61 & 1.06 & 0.78 & -1.44 & 1.56 \\
\hline & Bowel & -3.19 & -0.68 & 13.1 & 15.5 & 0.16 & -0.53 & 17.6 & 3.34 & -0.87 & -2.46 & 4.20 & 7.99 \\
\hline \multirow{4}{*}{ average $3 \mathrm{D}$ gamma } & PTV & 0.50 & 0.40 & 0.18 & 0.28 & 0.41 & 0.44 & 0.42 & 0.26 & 0.33 & 0.33 & 0.36 & 0.10 \\
\hline & Rectum & 0.33 & 0.27 & 0.20 & 0.21 & 0.34 & 0.14 & 0.27 & 0.15 & 0.12 & 0.27 & 0.23 & 0.08 \\
\hline & Bladder & 0.40 & 0.34 & 0.12 & 0.24 & 0.41 & 0.26 & 0.23 & 0.18 & 0.06 & 0.23 & 0.25 & 0.11 \\
\hline & Bowel & 0.28 & 0.23 & 0.11 & 0.18 & 0.26 & 0.18 & 0.2 & 0.25 & 0.19 & 0.20 & 0.21 & 0.05 \\
\hline$\%$ difference in D95 & PTV & 0.34 & -0.41 & 0.23 & 0.07 & -0.20 & -0.64 & -2.43 & -0.78 & -0.43 & -1.43 & -0.57 & 0.84 \\
\hline
\end{tabular}

\section{VMAT plans}

In Table 2, $10 \mathrm{H} \& \mathrm{~N}$ patients percentage difference of average dose, percentage difference of D95 and D1 and average 3D gamma between Eclipse TPS calculated and COMPASS measured for PTV's and critical OAR's were listed. Also, percentage variation of point dose measurement and GAI was listed. Table 3 summarizes the percentage difference of average dose, percentage difference of D95 and average 3D gamma for PTV, rectum, bladder and bowel for 10 pelvis patients.

\section{Discussion}

Advantage of COMPASS 3D dosimetry system over other QA systems is its capability of performing 3D dose reconstruction on patient CT scan using beam modeling, detector measurement and treatment plan. The dose calculation algorithm in an inhomogeneous medium was completely different in Eclipse TPS and COMPASS 3D dosimetry system. The COMPASS uses CCC/S algorithm, where the dose calculation is based primarily on a point source dose spread array. The Eclipse TPS uses AAA wheredose calculation is based on a pencil beam in association with lateral density scaling. In CCC/S, the dose at a point from a point source of given TERMA (total energy released per unit mass) to the dose at another location in a patient can be calculated by scaling both primary and scatter. Point to point density scaling of this kind is not feasible by the pencil beam kernel method. The point spread kernel based method allows greater flexibility in dealing with 3D inhomogeneous medium than pencil beam kernel. Due to this, maximum average dose difference of $3.2 \%$ in air was observed. ${ }^{14,20}$ Open field measurements provide vital information for fine tuning of beam 
modeling and help to understand the efficiency of dose reconstruction, especially in the buildup, air and penumbra region, so that one can set the tolerance level when OAR falls in region of interest. For example, in $H \& N$ patients lips lie in surface (build-up), trachea lies in air and cochlea lies in the field border (penumbra).

For PTV in Table 1, there was a systematic decrease in GAI and increase in average $3 \mathrm{D}$ gamma with respect to increase in treatment delivery errors. However, 2D planar dosimetry does not provide any clinically relevant information about the results, ${ }^{12}$ whereas COMPASS system provides the significance of error in PTV and as well as in OAR's. The information provided by traditional $1 \mathrm{D}$ point dosimetry and $2 \mathrm{D}$ planar devices cannot easily be translated onto dose deviations in the tumor and/or at OAR's. DVH based evaluation will be a good alternative since it allows physicist and physician to accept or reject the treatment plan based on the dose difference in PTV and OAR's. Measurements based dose reconstruction of COMPASS enables us to verify the treatment delivery with acceptable accuracy $( \pm 5 \%)$. PTV in Table 1 shows there was a systematic increase and decrease in the percentage difference of average dose with respect to known dose delivery errors, and an increase in average 3D gamma with planned MLC errors. ${ }^{16,21}$ In the COMPASS system, the difference between predicted and measured response is into two components. ${ }^{16}$ The first component is a scaling correction (Acorr) factor, which is used to find dose errors (incorrect $\mathrm{MU}$ ) and the second component is the remaining residual response, which is used to reconstruct MLC errors. The calculation grid resolution used in the TPS and COMPASS affects the accuracy of the dose distribution calculated. Literatures have reported that a $2.5 \mathrm{~mm}$ isotropic grid produces an accuracy of about $1 \%$ in the high-dose gradient region of an IMRT plan consisting of multiple fields. ${ }^{23}$ Benedict et al. ${ }^{23}$ and Park et al. ${ }^{24}$ have recommend grid size less than $3 \mathrm{~mm}$ for stereotactic treatments. In both Eclipse TPS and COMPASS QA system grid size resolution can be varied from 1 $\mathrm{mm}$ to $5 \mathrm{~mm}$, for our study we have chosen $2.5 \mathrm{~mm}$ grid size. Although smaller grid size can yield more accurate results, $2.5 \mathrm{~mm}$ grid size was chosen by optimizing the accuracy and computational time.

The results show that $3 \mathrm{D}$ dose parameters were well within the clinically acceptable tolerance level of $\pm 5 \% .{ }^{22}$ The average 3D gamma for PTV's and OAR's for twenty patients used in this study were less than the recommended value of 0.6 by Visser et al. ${ }^{17}$ In three pelvis cases due to low dose of bowel, the percentage difference of average dose was more than $10 \%$. DVH in COMPASS provides many statistical tools; however, we cannot judge plan quality by choosing one criteria, as the percentage difference in average dose, dose at volume and volume at dose will be too high for structure lying in low dose region. For higher deviation of fluence over small distances there are limitations due to the reconstruction capabilities of COMPASS and chamber resolution of MatriXX ${ }^{\text {Evolution. }}$. So COMPASS may slightly underestimate and/oroverestimate the actual delivered dose for OAR's. ${ }^{14}$ Despite local inaccuracies in the dose reconstruction, Godart et $a .^{16}$ have proven that COMPASS can be used to perform pretreatment verification of IMRT treatment plans. COMPASS can compute dose in patient CT scan using only inbuilt beam model (without measurements). This model based dose calculation, function as an independent secondary TPS verification. COMPASS have dose calculation engine (CCC/S) similar to TPS, it not only verify the plan parameters (MU) but also provides anatomically localized QA dose information. Kunnanchath et al. ${ }^{25}$ have showed good agreement in comparison of Eclipse TPS calculated vs COMPASS calculated with average dose difference less than $1 \%$ and average gamma less than 0.5 for PTV and OAR's of 10 head and neck and 10 pelvis IMRT plans. Previous studies have shown that measurement (MatriXX ${ }^{\text {Evolution }}$ ) based dose reconstruction in phantom images were in excellent agreement with the ion chamber results and planar dosimetry (film). ${ }^{13-15}$ Our study shows that COMPASS is a better system than point and 2D planar dosimetry by providing the $3 \mathrm{D}$ dose discrepancies in the region of interest in patient anatomy.

\section{Conclusion}

Traditionally QA methods are performed in a phantom and it is often difficult to quantify and interpret the results in patient anatomy. The ability of the COMPASS software to reconstruct the $3 \mathrm{D}$ dose distribution on patient CT from the measurements, provides a unique perspective for medical physicist and radiation oncologist to evaluate the patient's QA plan. The results from this study show that the COMPASS along with MatriXX ${ }^{\text {Evolution }}$ can be effectively used for pretreatment verification of VMAT plans and it is good enough to find clinical significant errors in dose delivery.

\section{Conflict of interest}

The authors declare that they have no conflicts of interest. The authors alone are responsible for the content and writing of the paper.

\section{References}

1. Vanetti E, Clivio A, Nicolini G, et al. Volumetric modulated arc radiotherapy for carcinomas of the oro-pharynx, hypo-pharynx and larynx: a treatment planning comparison with fixed field IMRT. Radiother Oncol 2009; 92:111-7.

2. Rana S, Pokharel S, Zheng Y, et al. Treatment planning study comparing proton therapy, RapidArc and intensity modulated radiation therapy for a synchronous bilateral lung cancer case. Int $J$ Cancer Ther Oncol 2014; 2:020216. 
3. Subramanian S, Srinivas C, Ramalingam K, et al. Volumetric modulated arc-based hypofractionated stereotactic radiotherapy for the treatment of selected intracranial arteriovenous malformations: dosimetric report and early clinical experience. Int J Radiat Oncol Biol Phys 2012; 82:1278-84.

4. Ezzell GA, Galvin JM, Low D, et al. Guidance document on delivery, treatment planning, and clinical implementation of IMRT: report of the IMRT Subcommittee of the AAPM Radiation Therapy Committee. Med Phys 2003; 30:2089-115.

5. Dong L, Antolak J, Salehpour M, et al. Patient-specific point dose measurement for IMRT monitor unit verification. Int J Radiat Oncol Biol Phys 2003; 56:867-77.

6. Masi L, Casamassima F, Doro R, Francescon P. Quality assurance of volumetric modulated arc therapy: Evaluation and comparison of different dosimetric systems. Med Phys 2011; 38: 612-22.

7. Herzen J, Todorovic M, Cremers F, et al. Dosimetric evaluation of a $2 \mathrm{D}$ pixel ionization chamber for implementation in clinical routine. Phys Med Biol 2007; 52:1197-208.

8. Fenoglietto P, Laliberte B, Ailleres N, et al. Eight years of IMRT quality assurance with ionization chambers and film dosimetry: experience of the Montpellier Comprehensive Cancer Center. Radiat Oncol 2011; 6:85.

9. Charland PM, Chetty IJ, Yokoyama S, Fraass BA. Dosimetric comparison of extended dose range film with ionization measurements in water and lung equivalent heterogeneous media exposed to megavoltage photons. J Appl Clin Med Phys 2003; 4: 25-39.

10. Howell RM, Smith IP, Jarrio CS. Establishing action levels for EPID-based QA for IMRT. J Appl Clin Med Phys 2008; 9: 16- 2721.

11. Nicolini G, Vanetti E, Clivio A, et al. The GLAaS algorithm for portal dosimetry and quality assurance of RapidArc, an intensity modulated rotational therapy. Radiat Oncol 2008; 3:24.

12. Nelms BE, Zhen H, Tomé WA. Per-beam, planar IMRT QA passing rates do not predict clinically relevant patient dose errors. Med Phys 2011; 38:1037-44.

13. Boggula $\mathrm{R}$, Jahnke $\mathrm{L}$, Wertz $\mathrm{H}$, et al. Patient-specific 3D pretreatment and potential 3D online dose verification of Monte Carlo-calculated IMRT prostate treatment plans. Int J Radiat Oncol Biol Phys 2011; 81:1168-75.

14. Boggula R, Lorenz F, Mueller L, et al. Experimental validation of a commercial $3 \mathrm{D}$ dose verification system for intensity-modulated arc therapies. Phys Med Biol 2010; 55:5619-33.

15. Korevaar EW, Wauben DJ, van der Hulst PC, et al. Clinical introduction of a linac head-mounted 2D detector array based quality assurance system in head and neck IMRT. Radiother Oncol 2011; 100:446-52.

16. Godart J, Korevaar EW, Visser R, et al. Reconstruction of high-resolution 3D dose from matrix measurements: error detection capability of the COMPASS correction kernel method. Phys Med Biol 2011; 56:5029-43.

17. Visser R, Wauben DJ, de Groot M, et al. Efficient and reliable $3 \mathrm{D}$ dose quality assurance for IMRT by combining independent dose calculations with measurements. Med Phys 2013; 40:021710.

18. Venkataraman S, Malkoske $\mathrm{KE}$, Jensen $\mathrm{M}$, et al. The influence of a novel transmission detector on 6 MV x-ray beam characteristics. Phys Med Biol 2009; 54:3173-83.

19. Fraass B, Doppke K, Hunt M, et al. American Association of Physicists in Medicine Radiation Therapy Committee Task Group 53: quality assurance for clinical radiotherapy treatment planning. Med Phys 1998; 25:1773-829.

20. Hasenbalg F, Neuenschwander H, Mini R, Born EJ. Collapsed cone convolution and analytical anisotropic algorithm dose calculations compared to VMC++ Monte Carlo simulations in clinical cases. Phys Med Biol 2007; 52:3679-91.

21. Fredh A, Scherman JB, Fog LS, Rosenschöld PM. Patient QA systems for rotational radiation therapy: a comparative experimental study with intentional errors. Med Phys 2013; 40:031716.

22. Thwaites D. Accuracy required and achievable in radiotherapy dosimetry: have modern technology and techniques changed our views? J Phys 2013; 444: 012006.

23. Park JY, Kim S, Park HJ, et al. Optimal set of grid size and angular increment for practical dose calculation using the dynamic conformal arc technique: a systematic evaluation of the dosimetric effects in lung stereotactic body radiation therapy. Radiat Oncol 2014; 9:5.

24. Benedict SH, Yenice KM, Followill D, et al. Stereotactic body radiation therapy: the report of AAPM Task Group 101. Med Phys 2010; 37:4078-101.

25. Kunnanchath J, Majumdar SKD, Tharmarnadar G, et al. Validation of compass QA system and the independent verification of dose calculation algorithm for IMRT patient specific plans. Transworld Medical Journal 2014; 2:170-5. 